\title{
On the Effects of the Excess Bias of the SPAD on the Timing Accuracy in Time Interval Measurement
}

\author{
J. Nissinen, I. Nissinen, J. Huikari, S. Jahromi, J.-P. Jansson and J. Kostamovaara \\ University of Oulu, Circuits and Systems Research Unit \\ Oulu, Finland \\ jan.nissinen@oulu.fi
}

\begin{abstract}
The timing accuracy of a single-photon avalanche diode (SPAD) based receiver is analyzed as a function of excess bias voltage. The width of the used optical pulse was $100 \mathrm{ps,} \mathrm{which}$ matches well with the jitter of the used SPAD receiver fabricated in a $0.35 \mu \mathrm{m}$ HVCMOS technology. The timing error was measured to be $900 \mathrm{ps}$ within the excess bias voltage range of $\sim 1.25$ $\mathrm{V}$ - 3.25 V. The single-shot resolution changes from 420 ps to 160 ps (FWHM), respectively.
\end{abstract}

\section{Keywords - Jitter, Timing error, Geiger detector}

\section{INTRODUCTION}

The use of single-photon avalanche diodes (SPAD) or diode arrays integrated together with a time interval measurement unit has increased a lot within the last ten years. One enabling fact is that SPADs can be fabricated in standard complementary metaloxide semiconductor (CMOS) technologies [1]. This kind of combination can be used in many applications as a timecorrelated single-photon counting unit as, for example, in fluorescence lifetime imaging, time-of-flight (TOF) laser range imaging, positron emission tomography, and time-gated Raman spectroscopy [2-4].

All of the mentioned applications require sub-nanosecond accuracy in time domain to achieve proper performance from the application point of view. In TOF laser range imaging, for example, the cm-level accuracy corresponds to $67 \mathrm{ps}$ in time domain. The SPAD itself is basically an avalanche photo diode (APD) which has been reverse-biased over the breakdown voltage in Geiger mode. The inherent timing resolution of the SPAD is approximately in the range of 50 - 100 ps when fabricated in CMOS technologies, which matches well with the requirements of applications. Moreover, the SPAD can be defined as a digital component which gives a logic level signal at the output when illuminated even by a single photon. All of these features of SPAD make it suitable for integrated timecorrelated single-photon counting sensors to be used in many applications. In addition, the time-to-digital converter should have proper accuracy and precision to measure the time-ofarrival of single photons accurately. Nowadays, TDCs integrated together with SPADs can offer a few tens of picoseconds' accuracy, which matches well with the jitter of SPAD [3,5].

Even though $100 \mathrm{ps} / \mathrm{cm}$-level accuracy can be realized in theory, there are error sources which can produce systematic and random timing inaccuracies in the SPAD-based time-correlated single-photon counting unit such as a walk error caused by the multiple-photon operation and the finite pulse width of the used laser source [6]. The change of the excess bias, the additional voltage over the breakdown voltage of a SPAD, can also cause timing error, which can be significant compared to the required accuracy, when a simple digital logic receiver is used together with a SPAD. The scale of this inaccuracy depends on the level of an excess bias of the SPAD, in which case any drift or ringing of the excess bias might cause the significant degrading of the precision and the accuracy of a time domain measurement. For example, in triggered SPAD imaging the widest dynamic range is achieved by using as low excess bias as possible because the dark count rate of a SPAD increases more rapidly than the photon detection efficiency by increasing the excess bias [7]. Then again, the maximum available excess bias is desirable in a TOF laser rangefinding where background noise is always present and an echo signal has to be maximized.

In this work, the effect of the excess bias voltage variation of the SPAD on timing accuracies was investigated by using the pulsed TOF lidar prototype with a 9x9 SPAD array [5] fabricated in $0.35 \mu \mathrm{m}$ HVCMOS technology and a laser diode transmitter with an optical pulse width of $100 \mathrm{ps}$. The aim was to get some practical information how regulated a high voltage supply would be needed to achieve a prober timing performance.

\section{PULSED TOF MEASUREMENT SETUP}

The pulsed TOF setup shown in Fig. 1 and used here is based on a (LD) laser diode [8] with a driver capable of producing an energetic (>1nJ), short (120 ps FWHM) laser pulse. The laser transmits optical pulses to a target, and reflected photons are collected by a 9x9 SPAD array with an on-chip 10-channel timeto-digital converter (TDC) having $10 \mathrm{ps}$ single-shot precision [5]. The jitter of SPADs (70 ps) matches well with the laser pulse width resulting in approximately $140 \mathrm{ps}$ single-shot precision for the whole TOF laser rangefinder because the other jitters sources in a circuit can be assumed to be negligible. The TDC is measuring the time intervals between an electrical start signal generated by a laser transmitter and 9 stop signals from the selected 3x3 SPADs of a 9x9 SPAD array. The optics are designed so that photons reflected from the target and entering the SPAD array are fit to the area of $3 \times 3$ SPADs. Distance has some effect on the position of the spot (spot size within $3 \times 3$ SPAD) in a SPAD array, but this can be observed from measurement results, and the new set of $3 \times 3$ SPAD can be selected when needed.

\section{SPAD FRONT END OPERATION}

The schematic and timing diagrams of a SPAD front end are shown in Fig. 2 a) and b). As mentioned above, the 3x3 SPADs

This work was supported by the Academy of Finland, Centre of Excellence in Laser Scanning Research under Contract 272196, and Contracts 282405 and 292609. 
are selected to occupy nine channels of a TDC, and one channel is used for a start signal. This selection is made before measurements by using the signal $\mathrm{V}_{\text {Sel }}$ in Fig. 2 a) to enable a quenching and loading signal (here only one SPAD with the front end circuit is shown for clarity) as well as a tri-state output buffer. A SPAD is based on the structure presented in [1] and the breakdown voltage of it is $\sim 19 \mathrm{~V}$. To bias the SPAD in Geiger mode to detect single photons, the $\mathrm{V}_{\mathrm{HV}}$ has to be above the breakdown voltage $(19 \mathrm{~V})$ when the photon counts are read from an anode node. In that case, the excess bias of the SPAD (additional voltage over the breakdown) is $\mathrm{V}_{\mathrm{HV}}-19 \mathrm{~V}$, and thus the maximum high voltage $\mathrm{V}_{\mathrm{HV}}$ is limited to below $19 \mathrm{~V}+3.3$ $\mathrm{V}$ when the supply voltage of $3.3 \mathrm{~V}$ is used for inverters and a front end. In that case, the quenching transistor M2 can quench the SPAD when needed, as explained in more detail below.

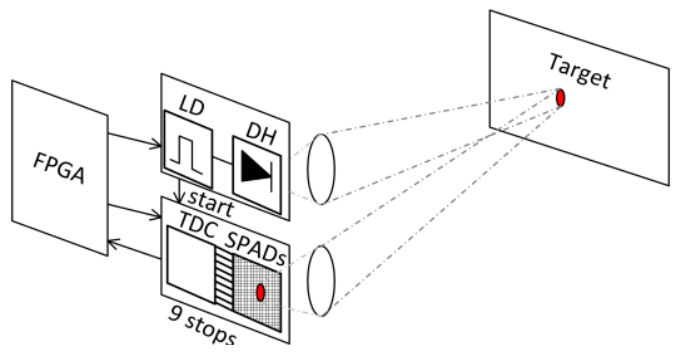

Fig. 1. Principle of TOF with SPAD array and 10-channel TDC.

At the beginning of a measurement cycle all of the selected SPADs are kept quenched by the signal $\mathrm{V}_{\text {Quench }}$ at ground. After that the time interval measurement is started by a start signal from the laser, and the TDC generates the rising edge of the $\mathrm{V}_{\text {Quench }}$ signal in Fig. 2. The anode node starts to float and has still the voltage of $3.3 \mathrm{~V}$, in which case the voltage over the SPAD is below the breakdown. After that the anode node is discharged to ground through a transistor M1 by the short positive $\mathrm{V}_{\text {Load }}$ pulse generated by the TDC. Now the SPAD is ready to detect single photons. When a photon is detected by a $\mathrm{SPAD}$, the voltage of the anode node $\left(\mathrm{V}_{\text {anode }}\right.$ in Fig. 2$)$ increases until the SPAD is quenched. The first inverter connected at the anode node is sized to have the threshold of approximately $1 \mathrm{~V}$ ( $\mathrm{V}_{\text {th }}$ in Fig. 2). After the range of the TDC is exceeded, the SPADs which weren't triggered by a photon or noise are quenched by the falling edge of the $\mathrm{V}_{\text {Quench }}$ signal from the TDC, and the next measurement cycle can begin.

Note that when the excess bias is below $3.3 \mathrm{~V}$, the amplitude of the anode node is limited more or less to the value of excess bias when triggered by a photon or noise, because avalanche breakdown is self-quenched by the increasing voltage and thus decreasing avalanche current. The excess bias of the SPAD is controlled by adjusting $\mathrm{V}_{\mathrm{HV}}$. For example, if the excess bias of $1 \mathrm{~V}$ was used, the $\mathrm{V}_{\mathrm{HV}}$ should be $20 \mathrm{~V}$ and thus the anode node will increase from zero to $1 \mathrm{~V}$ when triggered by a photon and quenching the SPAD because the voltage above the SPAD is less than $19 \mathrm{~V}$.

\section{Simulations AND MEASUREMENTS}

From the point of view of TOF laser rangefinding measurement it is very important to know the timing moment variation as a function of the signal amplitude, i.e. excess bias voltage. As mentioned in the introduction, the $\mathrm{cm}$-level distance measurement accuracy allows a timing error of $~ 100$ ps only in a timing discrimination circuit. Thus, a simulation was carried out to predict the timing error and then a measurement was made to compare the results to each other.

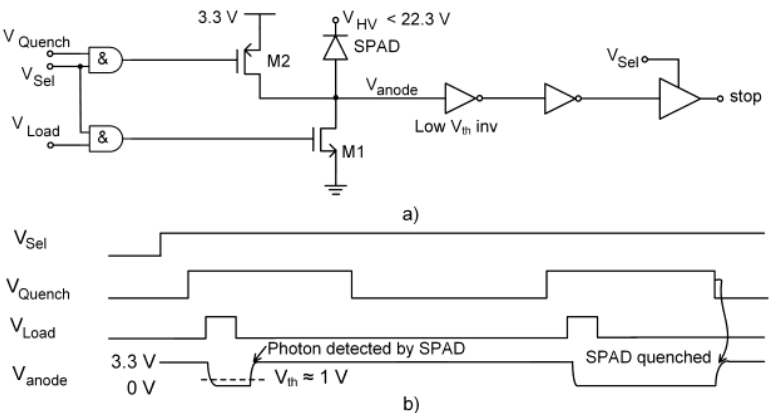

Fig. 2. a) Schematic and b) timing diagrams of a SPAD front end.

\section{A. Simulation model}

The SPAD can be modelled as a parallel resistor and capacitor in series with an ideal switch [9]. To evaluate the rise time of the anode node $V_{\text {anode }}$ (shown in Fig. 2.), when a photon is detected, also parasitic capacitances at the anode have to be considered. As presented in [9], the time constant defining the rise time of the avalanche process is dependent on the total capacitance at the anode and the dynamic resistance of the SPAD $\left(R_{d}\right)$. The dynamic resistance of the SPAD $\left(R_{d}\right)$ is formed by the sum of the space-charge resistance and the resistance of the neutral regions crossed by the avalanche current. The total capacitance of the anode was approximated according to the post-layout simulation (300 fF) and the dynamic resistance was measured to be $\sim 500 \Omega$ by a curve tracer which causes the anode time constant $\left(\tau_{\mathrm{an}}\right)$ of 150 ps so that the rise time of $2.2^{*} \tau_{\mathrm{an}}$ is produced when a step response is considered for the avalanche signal. Thus, the timing error of $\Delta \mathrm{t}_{1}-\Delta \mathrm{t}_{3}$ is generated between the minimum and maximum signal, as shown in Fig. 3. So even in an ideal comparator with a high bandwidth and maximum gain the timing walk of $\sim 200$ ps can be produced by the time constant of the anode. In addition, the post layout simulation was carried out to approximate the time error caused by the propagation delay variation of the low threshold inverter (Low $\mathrm{V}_{\text {th }}$ inv in Fig. $2 \mathrm{a}$ )) at the different overdrive voltages, i.e. excess bias voltages. The simulation showed that approximately a total error of 1200 ps was generated when excess bias was swept from $1.25 \mathrm{~V}$ to $3.3 \mathrm{~V}$ as shown in Fig. 4 (grey curve).

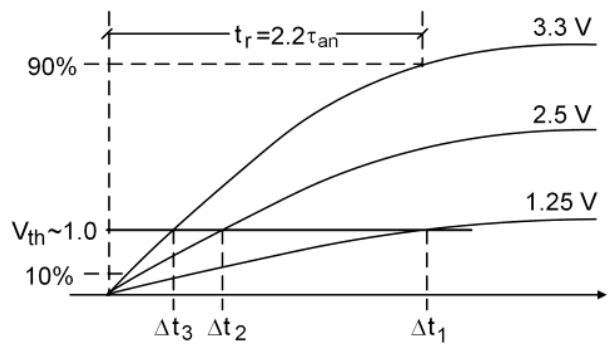

Fig. 3. A timing error generated by the time constant of the anode.

\section{B. TOF measurement as function of excess bias}

TOF measurements were made by changing the excess bias voltage $\left(\mathrm{V}_{\mathrm{EX}}\right)$ from $1.25 \mathrm{~V}$ to $3.3 \mathrm{~V}$ and collecting the time 
domain photon distribution of 560000 pulses transmitted to the target. The distance of the target was approximately $5.8 \mathrm{~m}$ in all measurements. With all excess biases, the photon detection probability was kept below $10 \%$ to guarantee that SPADs are working in the single photon mode. The normalized time domain photon distributions at the different excess biases are shown in Fig. 5. As can be seen in Fig. 5 at the excess bias voltage of $1.25 \mathrm{~V}$ (rightmost distribution), the full width half maximum value of the distribution is wider compared to those of distributions with a larger excess biases. Thus, the jitter at the timing discriminator (inverter) starts to affect the deviation of the total distribution. The full width half maximum value at the excess bias of $3.25 \mathrm{~V}$ is approximately $150 \mathrm{ps}$.

The timing error as a function of excess bias was calculated from the peak values of the hit distribution and has been presented in Fig. 4 (black curve). The total timing error was approximately $900 \mathrm{ps}$, resulting to $135 \mathrm{~mm}$ in distance. In other words, the timing dependence as a function of excess bias within the full dynamic range of the inverter was $450 \mathrm{ps} / \mathrm{V}$. Fortunately, the timing error is mostly caused by the excess biases of $1.25 \mathrm{~V}$ $-2 \mathrm{~V}$, so for example from an excess bias of $2.4 \mathrm{~V}$ to $3.25 \mathrm{~V}$, the timing error of $12 \mathrm{ps} / 100 \mathrm{mV}$ is caused.

The total timing error of 900 ps caused by the excess bias voltage cannot be explained only by the geometrical timing error dependent on the rise time of the signal at the input of the timing discriminator. However, according to the post layout simulation of the circuit, most of the timing walk was caused by the delay variation of the inverter depending on the different signal amplitude or the overdrive of the inverter threshold [10]. As can be seen in Fig. 4 the simulated timing error has a steeper slope at the smaller excess biases when compared to the measured error. This can be explained by the process parameter variation which has a high influence to the threshold and the driving capability of an inverter and thus causing a larger uncertainty for approximation of the delay with the minimum overdrive.

\section{CONCLUSIONS}

The timing error dependent on the excess voltage of the SPAD is important in every time of arrival or time-correlated single photon counting based measurement applications, such as Raman spectroscopy and pulsed time-of-flight laser radars. In these applications, an error of only tens of picoseconds can be crucial when high accuracy is required. Here the pulsed TOF lidar based on a SPAD array fabricated on $0.35 \mu \mathrm{m}$ HVCMOS technology and a quantum well laser diode having an optical pulse width of $100 \mathrm{ps}$ have been evaluated from the timing error point of view produced by the excess bias voltage of the SPAD. The measurements showed that a timing error of $450 \mathrm{ps} / \mathrm{V}$ occurs when the excess bias voltage is swept from $1.25 \mathrm{~V}$ to 3.25 V. However, the timing error is not directly proportional to the voltage but it experiences an exponential decaying function. Thus, the voltage dependence of the timing error on the last 850 $\mathrm{mV}$ was only $12 \mathrm{ps} / 100 \mathrm{mV}$, which is $1.8 \mathrm{~mm} / 100 \mathrm{mV}$ in distance.

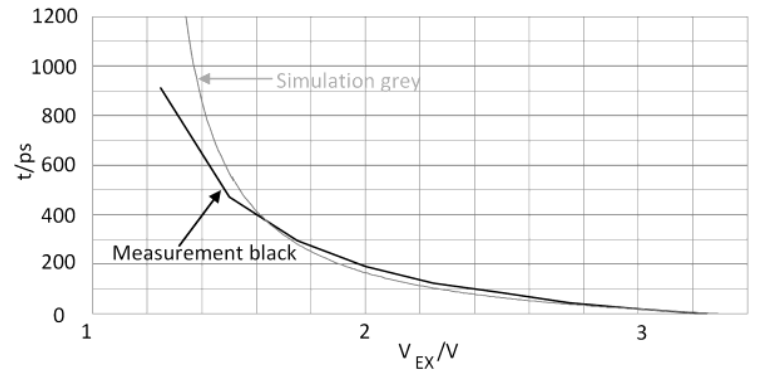

Fig. 4. The simulated (grey) and measured (black) timing error as function of excess bias voltage.

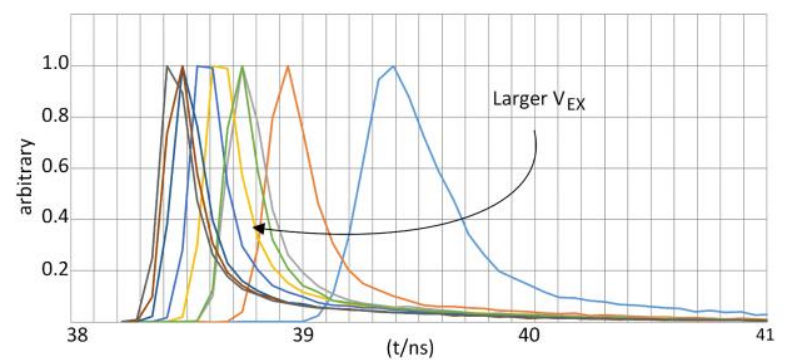

Fig. 5. The normalized hit distributions at the different excess biases from 1.25 to 3.25 with $0.25 \mathrm{~V}$ step.

\section{REFERENCES}

[1] A. Rochas et al., "Single Photon Detector Fabricated in CMOS High Voltage Technology", Review of Scientific Instrumnets, vol. 74, no. 7, pp. 3263-3270, 2003.

[2] C. Niclass, M. Soga, H. Matsubara, M. Ogawa, M. Kagami, “A 0.18- $\mu \mathrm{m}$ CMOS SoC for a 100-m-Range 10-Frame/s 200 x 96-Pixel Time-ofFlight Depth Sensor," IEEE Journal of Solid-State Circuits, vol. 49, no. 1, pp. 315-330, Jan. 2014.

[3] L. H. Brage et al.“ An 8x16-pixel 92 kSPAD time-resolved sensor with on-pixel $64 \mathrm{ps} 12 \mathrm{~b}$ TDC and $100 \mathrm{MS} / \mathrm{s}$ real-time energy histogramming in $0.13 \mu \mathrm{m}$ CIS technology for PET/MRI Applications," in IEEE Int. Solid-State Circuit Conference Dig. Tech . Papers, pp. 486-487, Jan. 2013.

[4] I. Nissinen et al. "A sub-ns time-gated CMOS single photon avalanche diode detector for Raman spectroscopy", in Proc. IEEE ESSDERC, pp. 375-378, 2011.

[5] S. Jahromi, J. Jansson, I. Nissinen, J. Nissinen and J. Kostamovaara, ”A Single Chip Laser Radar Receiver with a 9x9 SPAD Detector Array and a 10-Channel TDC," Proceedings of the ESSCIRC'15, Graz, Austria, pp. 364-367, Sep. 14-18, 2015.

[6] G. Kircher, F. Koidl, J. Blazej, K. Hamal and I. Prochazka, "Time-walkcompensated SPAD: multiple-photon versus single-photon operation," Proc. SPIE 3218, Laser Radar Ranging and Atmospheric Lidar Techniques, 106, December 22, 1997.

[7] E. Vilela et al."A low-noise time-gated single-photon detector in a HVCMOS technology for triggered imaging," Sensors and Actuators A: Physical, Vol. 201, pp. 342-351, 2013.

[8] J.M.T. Huikari et al. ," High-Energy Picosecond Pulse Generation by Gain Switching in Asymmetric Waveguide Structure Multiple Quantum Well Lasers," IEEE journal of Selected Topics in Quantum Electronics, vol. 21, no. 6, Art. no.1501206, Nov./Dec. 2015.

[9] A. Dalla Mora, A. Tosi, S. Tisa and F. Zappa,"Single-Photon Avalanche Diode Model for Circuit Simulation," IEEE Photonics Technology Letters, vol. 19, no. 23, pp.1922-1924, Dec. 2007.

[10] P. Palojärvi, T. Ruotsalainen and J. Kostamovaara," A 250-MHz BiCMOS receiver channel with leading edge timing discriminator for a pulsed time-of-flight laser rangefinder," IEEE Journal of Solid-State Circuits, vol. 40, no. 6, pp. 1341-1349, June 2005. 